\title{
eHEALTH: Redefining Health Care in the Light of Technology
}

\author{
Mike W. Chiasson \\ Lancaster University, Lancaster, U.K. \\ Donal Flynn \\ University of Manchester, Manchester, U.K. \\ Bonnie Kaplan \\ Yale University, New Haven, CT U.S.A. \\ Pascale Lehoux \\ Université de Montréal, Montreal, Canada \\ Cynthia LeRouge \\ St. Louis University, St. Louis, MO U.S.A.
}

\section{INTRODUCTION}

eHealth is the use of emerging Information and Communication Technology, especially the Internet, to improve or enable health and healthcare. (Eng 2004)

Information and communication technology is now the major enabler for healthcare organizations on many levels- national, regional and local-hoping to achieve structural and cultural change in healthcare provision; for example, the UK's NPfIT (National Program for IT) and the National Health Information Initiative in the United States. Final NPfIT costs are variously estimated from $£ 12$ billion to $£ 31$ billion. Major initiatives are also underway in other developed as well as developing economies to address healthcare issues with eHealth technologies.

Please use the following format when citing this chapter:

Chiasson, M., Flynn, D., Kaplan, B., Lehoux, P., and LeRouge, C., 2008, in IFIP International Federation for Information Processing, Volume 267, Information Technology in the Service Economy: Challenges and Possibilities for the $21^{\text {st }}$ Century, eds. Barrett, M., Davidson, E., Middleton, C., and DeGross, J. (Boston: Springer), pp. 357-362. 
There are four main drivers of change in healthcare today:

- the new consumer (e.g., demanding information, control, choice, and service)

- the new science (of evidence-based medicine)

- the new technology (e.g., the Internet, monitoring devices and sensors, smart phone applications)

- the new focus on quality (e.g., the reduction of medical mistakes, comparative performance measurement, and focus on outcomes)

The drivers are combining to place new demands on healthcare organizations and providers who are faced with developing more efficient and effective healthcare delivery systems in the face of challenges such as ageing populations, who are expecting an extended life expectancy to be of high quality, and worldwide increase in chronic disease such as diabetes. Such systems are increasingly based on advances in ICT, changes in social demographics resulting in wider access to various technologies, and more informed patient demand. These changes are both contributing to and resulting in greater emphasis on community healthcare, patient self-management, and private healthcare provision. eHealth has thus both a technological as well as a social dimension, involving the use of ICT for health and a patient-centered approach to health.

While considerable advantages are expected for eHealth initiatives, these trends present both practice and research challenges. Considerable expertise is needed to design, develop, and evaluate ICT-based eHealth systems to suit a wide range of stakeholder perspectives in a variety of social contexts and changing patterns of health care delivery. Few health administrators and even fewer healthcare providers have the required mix of technological and social expertise. Medical informatics, management, and information systems researchers, too, face challenges. eHealth systems pose an interesting challenge to an organization-centric view of interacting social and technological systems. The different values and perceptions that individuals bring to bear on the patient-provider relationship, and the identification of social and cultural influences on patients' health beliefs and behaviors, including the use of ICT for eHealth purposes, pose policy, design, and deployment challenges. Another challenge is that, although qualitative methods are increasingly accepted, in many circles methodological standards in health care still regard the RCT (randomized control trial) as the gold standard and it is difficult to demonstrate that a particular element of an eHealth intervention, typically a complex, multi-element techno-social system, is a significant shaping influence on the chosen outcomes.

Thus, difficult and challenging issues arise as ICT is increasingly used in the expectation of coproducing health care and health care delivery. Examining what is occurring in healthcare points to emerging issues relevant to information systems researchers in other domains as well. This panel will address these issues from a variety of disciplinary and national perspectives by drawing implications for IS research from studies involving several different eHealth applications. A common theme is the nature and extent of change, individual and institutional, required for successful eHealth implementation.

Among the topics panelists will explore are:

- An overview of eHealth research areas, covering a range of stakeholder perspectives, with a focus on patients and healthcare providers. Advantages will be summarized, 
followed by a discussion of the evidence for improvements, leading to a summary of problems encountered in eHealth implementations.

- The redefinition of healthcare and its implications for patients (e.g., empowerment, culture, access) and the patient-provider relationship (e.g., nature of the relationship, provider education).

- The conflict between a range of institutional normative pressures and the values underlying eHealth initiatives, and the impact on evolving system designs.

- The extent and nature of changes required to traditional patient and provider roles and workflow and the different perspectives concerning a successful eHealth "encounter."

- From a technological innovation perspective, designers embed their assumptions about chronic illness into eHealth systems, and a narrow, efficiency-based managerial and clinical perspective can create as much as it can solve the problem of chronic illness.

\section{PANELISTS AND TOPICS}

Mike W. Chiasson is currently an Advanced Institute for Management Research (AIM) fellow and a senior lecturer in the Department of Management Science at Lancaster University's Management School. His research examines how social context affects IS development and implementation, using a range of social theories (actor network theory, structuration theory, critical social theory, ethnomethodology, communicative action, power-knowledge, deconstruction, and institutional theory). In studying these questions, he has examined various development and implementation issues (privacy, user involvement, diffusion, outsourcing, cyber-crime, and system development conflict) within medical, legal, engineering, entrepreneurial, and governmental settings. Mike can be reached at m.chiasson@lancaster.ac.uk.

Mike's discussion will focus on the various types of institutional pressures that affect development and use risks during eHealth initiatives aimed at reconfiguring patientprovider interaction (coproduction). His example will include the implementation of web-based systems to support communication between diabetes patients and their providers (nurses and physicians). While the institutional influences in health care are considered to some extent elsewhere, the timing and appearance of these influences and their management during eHealth projects, raise some potentially new insights. Four institutional pressures — hygienic, beneficial, effort-based, and equitable — will be discussed. He will also illustrate how the various eHealth initiatives were designed, shaped, and pitched in an attempt to address these institutional pressures.

Donal Flynn is a senior lecturer at the Manchester Business School and has research interests in the social and psychological processes that underlie the interaction between information systems and their human and organizational contexts. In 2005, he was a visiting research scholar in the Computer Information Systems Department, Robinson School of Business, Georgia State University in Atlanta. Currently, his focus is on eHealth, investigating interactive eHealth systems such as patient decision aids and webbased co-management systems for long-term conditions. Donal can be reached at donal.flynn@manchester.ac.uk. 
Donal will present an overview of the different eHealth research areas, outlining their relationship to the different levels of stakeholders such as patient, healthcare provider, primary, secondary, and tertiary care institutions, healthcare planning and commissioning authority, and national epidemiological research institute. An information therapy viewpoint will be taken for the patient perspective while the provider perspective will outline clinical decision support, electronic health record, and CPOE, professional education and infrastructure systems. Having set the scene, the advantages (both predicted and realized) of eHealth will be summarized, followed by a discussion of the existing evidence for improvements resulting from eHealth. This will lead to a summary of the problems encountered in eHealth implementation and some important issues raised. Finally, some interesting points relevant to the 8.2 community will be noted.

Bonnie Kaplan is a lecturer in Medical Informatics at the Yale School of Medicine and Yale College, and is an adjunct clinical professor of Biomedical and Health Information Sciences at the University of Illinois at Chicago. A Fellow of the American College of Medical Informatics and recipient of the American Medical Informatics Association President's Award, she is chair-elect of both the American Medical Informatics Association Working Groups on People and Organizational Issues and on Ethical, Legal, and Social Issues. Bonnie co-chaired the IFIP 8.2 conference resulting in the book Information Systems Research: Relevant Theory and Informed Practice (Kluwer, 2004). Her research interests include evaluation, organizational, social, and ethical issues concerning new technologies, especially in health care. Bonnie can be reached at bonnie.kaplan@yale.edu.

The coproduction of service in health care represents a change to increased patientcentric care and collaboration between providers and patients. It is also seen as a way of redefining health care through a global network of information and services that increases patient access, autonomy, and empowerment.

Bonnie's presentation draws on years of research and experience in medical informatics and information systems to reflect on the research and ethical issues arising from the redefinition of the patient/health care consumer role in light of these emerging systems and changing processes in health care. In particular, the following will be explored: (1) consumer issues, such as what constitutes consent, empowerment, and autonomy - who is being empowered to do what; (2) what affects access and use; (3) organizational issues of reengineering healthcare globally, such as structural changes and role changes in health care delivery; and (4) work-life issues, such as those involved in changes in the patient-provider relationship, and in health care workers' roles and skill levels. All of these have implications for information systems research in domains other than health care. They raise concerns about design, such as how to take account of differences among individuals, populations, national structures, and stakeholder viewpoints, as well as the values embedded in technology and design approaches; empowering consumers and workers; and research ethics.

Pascale Lehoux obtained her Ph.D. in Public Health from the University of Montreal (Quebec, Canada) in 1996. An associate professor in the Department of Health Administration and a researcher with the Groupe de Recherche Interdisciplinaire en Santé (GRIS) at the University of Montreal, Pascale currently holds a Canada Research Chair on Innovation in Health (2005-2010). Her CRC program examines upstream factors that have an impact on the ultimate use and dissemination of health technologies (e.g., the design process itself, including needs analyses, design strategies, market constraints and 
opportunities, and group perceptions and practices guiding the innovation processes). She has published more than 50 papers examining the use of computerized medical records, telemedicine, scientific knowledge, home care equipment, and mobile and satellite dialysis units. Routledge published her book, The Problem of Health Technology, in 2006. Pascale can be reached at pascale.lehoux@umontreal.ca.

Pascale's presentation critically explores the role of technological innovation in the constitution of chronic states and illness. Drawing on the co-construction of technology and society perspective, the presentation focuses specifically on the way innovation designers envisage the enhancement of the chronically ill and build certain kinds of sociotechnical configuration to deal with chronic illness.

Using the case of "intelligent distance patient monitoring" as an illustration, Pascale argues that technology creates as much as it solves the problem of chronic illness. Technology is recursively embedded in chronic illness and it generates dual effects: it constrains and sustains users' daily practices. In a context where lack of financial and human resources dominates, managerial and clinical aims are steering the design process in a way that may first and foremost seek efficiency and provide a narrow response to chronic patients' concerns and preferences. Only by recognizing technology's duality, and eventually transcending it, will research and policy initiatives be able to deal creatively and responsibly with the design of our future health experiences.

Cynthia LeRouge is an associate professor in the Decision Sciences and Information Technology Management Department at St. Louis University. Cynthia has held various management roles in practice including roles in the software and healthcare industries prior to joining academe. Her current research interests relate to health care information systems, and in particular telemedicine and consumer informatics. She has over 60 publications including academic journal articles, edited chapters in researchbased books, and peer-reviewed conference proceedings. Cynthia has co-chaired health care mini-tracks for various information systems conferences. She has also served as a special guest editor for the European Journal of Information Systems' special issue on Health Information Systems Research, Revelations and Visions. For the past year, she has actively worked as an executive board member of the Association for Information Systems special interest group for Healthcare Research and currently serves as the chair. Cynthia can be reached at lerougec@slu.edu.

There is a rising call in management literatures to revisit classic work design and process theories, given modern work technologies, practice, and roles in health care and other industries. Cynthia's presentation will cover the topic of coproducing new forms of technology-mediated service, specifically focusing on telemedicine (in the form of medical exams using high-end, real-time video conferencing and peripheral devices). These encounters may span the spectrum of contact from one-time exams with a specialist to enhanced encounters, where a patient and caregiver may meet via video conferencing on a periodic basis with multiple providers not colocated for the management of chronic diseases, such as AIDS. This phenomenon represents real-time coproduction of health service. Introducing technology as a mediator to the health delivery process initiates elements of change to the traditional roles of patients and providers, the medical exam workflow process, and the patient-physician communication process; ironically, it can also exacerbate some issues found in traditional service delivery. Although there is considerable overlap in what patients and providers define as key mechanisms that drive a successful medical video conferencing encounter process, there are differences 
that underscore the need for exploring multiple perspectives of health technologies in research and practice. The discussion will be based on a U.S. field study of medical video conferencing (telemedicine) and highlight the process, people, and mechanisms involved in coproducing the telemedicine encounter. 